\title{
Gradhiva
}

GRADHIV

Revue d'anthropologie et d'histoire des arts

$30 \mid 2019$

Précieux

\section{Arnaud Esquerre, Interdire de voir : sexe, violence et liberté d'expression au cinéma}

Paris, Fayard, coll. « Histoire de la pensée », 2019

\section{Jeanne Favret-Saada}

\section{OpenEdition}

\section{Journals}

Édition électronique

URL : http://journals.openedition.org/gradhiva/4758

DOI : $10.4000 /$ gradhiva. 4758

ISSN : 1760-849X

\section{Éditeur}

Musée du quai Branly Jacques Chirac

Édition imprimée

Date de publication : 4 décembre 2019

Pagination : 152-153

ISBN : 978-2-35744-113-2

ISSN : 0764-8928

\section{Référence électronique}

Jeanne Favret-Saada, « Arnaud Esquerre, Interdire de voir : sexe, violence et liberté d'expression au cinéma », Gradhiva [En ligne], 30 | 2019, mis en ligne le 31 mars 2021, consulté le 02 avril 2021. URL : http://journals.openedition.org/gradhiva/4758 ; DOI : https://doi.org/10.4000/gradhiva.4758

Ce document a été généré automatiquement le 2 avril 2021.

(c) musée du quai Branly 


\section{Arnaud Esquerre, Interdire de voir : sexe, violence et liberté d'expression au cinéma}

Paris, Fayard, coll. « Histoire de la pensée », 2019

Jeanne Favret-Saada

\section{RÉFÉRENCE}

Arnaud Esquerre, Interdire de voir : sexe, violence et liberté d'expression au cinéma, Paris, Fayard, coll. « Histoire de la pensée », 2019, 348 p. 


\section{Arnaud Esquerre}

\section{Interdire de voir}

Sexe, violence et liberté d'expression au cinéma

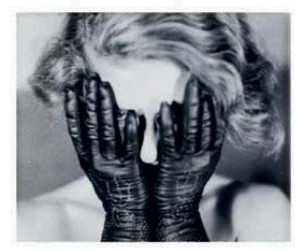

hdo

fayard

Depuis dix ans, Arnaud Esquerre a entrepris des enquêtes historiques et ethnographiques destinées à tester l'hypothèse générale d'un affaiblissement de l'État en France: or, plutôt qu'une extension indéfinie des libertés individuelles, il a enregistré le maintien ou même l'accroissement du contrôle de l'État sur les citoyens. Dans La Manipulation mentale: une sociologie des sectes et de la psychothérapie en France (Paris, Fayard, 2009), l'auteur a montré comment l'État protège désormais contre ellesmêmes des personnes en quête de mieux-être psychique par un nouveau dispositif de lutte contre les «sectes» et un délit de "manipulation mentale». Dans Les Os, les cendres et l'État (Paris, Fayard, 2011), il a examiné les mesures législatives et réglementaires par lesquelles l'État, outrepassant les nécessités de la santé publique, déboute les survivants de leur maîtrise sur leurs défunts et leur mémoire : les corps morts sont avant tout la propriété du territoire national et de la patrie. Le troisième volet de cette enquête sur l'État et le citoyen porte sur l'interdiction faite à certains spectateurs, ou à tous selon le cas, d'avoir accès aux films une fois produits. Dès lors, malgré le sous-titre de l'ouvrage, Interdire de voir concerne peut-être moins la liberté d'expression de l'artiste que le droit corrélatif du spectateur à prendre connaissance d'une œuvre filmique, le droit de «jouir des arts ${ }^{1}$ ». En exergue, l'auteur cite d'ailleurs l'universitaire allemand Victor Klemperer, évoquant en décembre 1938 "les derniers films que nous ayons eu encore le droit de voir » (c'est moi qui souligne). Même dans un régime aussi démocratique que celui de la France actuelle, l'État exerce un pouvoir de police administrative qui établit ce qui est moralement admissible au cinéma.

2 Partout dans le monde, les films sont l'ultime production artistique qui soit encore passible d'une censure a priori. Alors que les représentations théâtrales en ont été libérées depuis longtemps, la projection publique d'images animées et parlantes est tenue pour un spectacle potentiellement toxique, dont il convient de protéger certaines 
catégories de spectateurs ou bien la population tout entière. En France, la censure cinématographique est directement prise en charge par l'État en tant qu'il est le garant de l'ordre public et des "mœurs », et non, comme dans d'autres pays, par une instance indépendante du pouvoir exécutif, par exemple l'industrie cinématographique ellemême ou une association dotée d'une autorité morale: selon le mot de Dimitri Vezyroglou ${ }^{2}$, dans notre pays, le cinéma est une affaire d'État. Un film ne peut y être projeté sans que le ministre de la Culture, sur l'avis d'une commission d'examen des films, ne lui ait accordé une autorisation préalable, le visa d'exploitation, le refus de visa signifiant une interdiction totale ${ }^{3}$.

Dans Interdire de voir... comme dans ses deux ouvrages précédents, Arnaud Esquerre reconduit le modèle de l'enquête méthodique à la manière du XVIII ${ }^{\mathrm{e}}$ siècle, celle qui a précédé les partages disciplinaires du siècle dernier dans les sciences sociales. Il recourt en particulier à deux disciplines qui sont rarement convoquées dans les travaux de sociologie. L'histoire, tout d'abord, qui lui permet de montrer combien cet art récent qu'est le cinéma est aujourd'hui encore censuré selon des procédures conçues pour d'autres arts dès l'Ancien Régime, ou encore ce que la législation actuelle doit au régime de Vichy ${ }^{4}$. L'ethnologie ensuite, en assistant, pendant une année, aux réunions dans lesquelles s'élabore la "classification » des films, c'est-à-dire les avis destinés au ministre, qui leur donnera ou non l'autorisation d'être projetés dans des salles de cinéma ${ }^{5}$. La convergence des techniques d'enquête, notamment le recours à l'archive et l'analyse des débats en commission (" l'inarchivable »), donne à Interdire de voir... une richesse et une ampleur de perspectives peu communes dans ce genre d'ouvrages : on peut en effet le lire, au choix, comme un traité d'histoire de la censure, un essai de sociologie pragmatique sur la formation du jugement moral, une réflexion critique sur l'effet des images, l'ethnologie d'un corps social préposé à l'estimation morale des films, ou enfin comme un élément, susceptible d'agencement avec d'autres, d'une théorie générale de l'État dans son rapport avec les citoyens.

4 Le droit français du cinéma a toujours euphémisé l'activité étatique de censure en parlant de commissions de "contrôle ", ou de "classification ", alors qu'elles peuvent interdire totalement des films, et qu'elles l'ont fait pendant très longtemps. Arnaud Esquerre prend le parti de pulvériser ce déni, et d'employer, pour analyser l'activité de censure, un jeu de termes qui expriment cette éventualité : tout acte de censure sera une "suppression », pratiquée par des commissaires «suppresseurs ", afin de protéger la sensibilité morale de spectateurs « supprimés ${ }^{6}$ ». Or cette option radicale paraît en contradiction avec la réalité de la censure aujourd'hui, car l'ultime avatar de l'interdiction totale, le refus de visa, bien que présent dans le Code du cinéma et de l'image animée, n'a pas été infligé depuis quarante ans. Le Conseil d'État lui-même, enclin à désavouer le ministre de la Culture et à relever l'âge auquel il est licite d'assister à la projection de certains films, a toujours rejeté les demandes de refus de visa $^{7}$. Cet état de choses n'équivaut certes pas à une disparition pure et simple de la censure, mais l'on ne peut pas s'y référer comme à une "action" (p.12) puisque, précisément, rien de tel ne se produit. Par contre, il convient d'élucider la présence maintenue de cette éventualité légale, peut-être une menace qui opérerait sur le modèle de l'arme nucléaire.

5 Grâce à leur passé de consommateurs de films et de lecteurs de la presse, les lecteurs d'Interdire de voir... disposent déjà d'une certaine représentation de la censure cinématographique: or elle ne s'accorde guère avec les choix lexicaux d'Arnaud 
Esquerre. Il aurait pu signaler, par exemple que, depuis 1979, l'interdiction totale est impossible politiquement, sinon légalement; qu'au temps où elle se produisait, elle n'a jamais entraîné la destruction physique du film, à une exception près ${ }^{8}$; et enfin que, tôt ou tard, toutes les interdictions sont levées, si bien que les spectateurs "supprimés » ne le sont que pour un temps, celui où l'exécutif défend un certain ordre moral, avant de se raviser, parfois sur l'injonction du tribunal de l'abus de pouvoir, à savoir le Conseil d'État. Certes, ces considérations ne modifient pas la situation que l'auteur entend mettre en lumière, le fait que l'État français s'attribue le droit d'intervenir en matière de morale au cinéma, mais il aurait été bienvenu de pouvoir se représenter la réalité dont il est question.

Malgré l'inconfort que suscite l'appareil conceptuel de l'auteur, les parties centrales de l'ouvrage (II et III), qui portent sur l'activité de la commission de classification des œuvres cinématographiques, feront l'unanimité parmi les ethnologues et les partisans d'une sociologie pragmatique. Arnaud Esquerre a fait admettre sa présence à des débats qui lui étaient en principe fermés, et il a contourné avec élégance la consigne du secret administratif. Pendant plusieurs mois, il a noté les échanges entre les commissaires, il les a confrontés avec les dossiers des films en examen, et il les a éclairés par un grand nombre d'entretiens. Il montre ainsi comment l'État s'arroge le droit de restreindre la liberté de «jouir des arts» (et donc, celle de diffuser des images filmiques), tout en l'exerçant selon une procédure étrangement démocratique.

En effet, les commissaires sont choisis pour leur relation avec l'art et l'industrie du cinéma, ou avec cette catégorie sociale à protéger qu'est la jeunesse ; leurs convictions morales, bien que diverses, excluent néanmoins le rigorisme, puisque l'interdiction d'un film est hors de question. Esquerre montre excellemment selon quels principes chacun d'entre eux élabore sa propre interprétation d'un film problématique, et comment il la modifie ensuite, au fil du débat, afin qu'elle contribue à former « un acte collectif d'interprétation» (p. 107). Or ce sens mis en commun - l'avis de la commission - est déjà un texte juridique, puisque le ministre de la Culture le reprend à son compte sans le modifier : la signature ministérielle fait de cet avis une décision de l'exécutif, qui s'impose sur la totalité du territoire national. Ainsi l'État français, en conséquence de sa prétention à conformer la morale des citoyens, doit-il se résoudre, pour finir, à ne plus protéger que les plus faibles d'entre eux, et en s'appuyant sur un dispositif pseudodémocratique.

\section{NOTES}

1. Ce droit est garanti par l'article 27 de la Déclaration universelle des droits de l'homme de 1948, qui présuppose le droit à la liberté d'expression défini dans l'article 19. Lequel avait été proclamé dès 1789 dans l'article 11 de la Déclaration des droits de l'homme et du citoyen.

2. Le Cinéma : une affaire d'État, 1945-1970. Paris, La Documentation Française, 2014.

3. Le Code du cinéma et de l'image animée prévoit des sanctions particulièrement dissuasives en cas d'infraction. 
4. Parties I (« De la censure à la classification », depuis l'institution de la censure en 1916 jusqu'à nos jours) et IV («Une autre interprétation est possible», sur le durcissement de la censure depuis 2000).

5. Parties II («L'interprétation d'une œuvre sans son auteur») et III («Les spectateurs projetés »).

6. «Supprimer est entendu ici comme l'action imposée par des personnages que j'appelle des suppresseurs de faire disparaître, partiellement ou totalement, un film [...]. »; « [...] il est possible de supprimer un film en supprimant, partiellement ou totalement, son accès à des spectateurs, et c'est pourquoi je parlerai de spectateurs supprimables. » (Esquerre $2019: 12$ et 14)

7. En 2016, le représentant du ministère de l'Intérieur à la commission de classification a demandé sans succès qu'elle refuse un visa au documentaire Salafistes réalisé par Lemine Ould Mohamed Salem et François Margolin : la ministre a concédé un visa, assorti d'une interdiction aux moins de 18 ans. Le producteur a contesté cette décision devant le Conseil d'État, qui, en 2019, lui a donné raison.

8. En 1979, une coalition de militants catholiques et de défenseurs des valeurs familiales françaises a obtenu d'un tribunal que L'Essayeuse, un film "pornographique ", soit brûlé plutôt que d'être déposé à l'Enfer de la Bibliothèque Nationale (voir Jeanne Favret-Saada, Les Sensibilités religieuses blessées : christianismes, blasphèmes et cinéma, 1965-1988. Paris, Fayard, 2017 : 224-227).

\section{AUTEURS}

\section{JEANNE FAVRET-SAADA}

jfavretsaada[at]gmail.com 